\title{
3D numerical simulation of flow field with incompletely flaring gate pier in large unit discharge and deep tail water project
}

\author{
Zhou Zhao*, Wang Junxing \\ State Key Laboratory of Water Resources and Hydraulic Engineering Sciences, Wuhan University, Wuhan 430072, China
}

\begin{abstract}
Limited by large unit discharge above the overflow weir and deep tail water inside the stilling basin, the incoming flow inside stilling basin is seriously short of enough energy dissipation and outgoing flow still carries much energy with large velocity, bound to result in secondary hydraulic jump outside stilling basin and scour downstream river bed. Based on the RNG k- $\varepsilon$ turbulence model and the VOF method, this paper comparatively studies flow field between the conventional flat gate pier program and the incompletely flaring gate pier program to reveal energy dissipation mechanism of incomplete flaring gate pier. Results show that incompletely flaring gate pier can greatly promote the longitudinally stretched water jet to laterally diffuse and collide in the upstream region of stilling basin due to velocity gradients between adjacent inflow from each chamber through shrinking partial overflow flow chamber weir chamber, which would lead to large scale vertical axis vortex from the bottom to the surface and enhance mutual shear turbulence dissipation. This would significantly increase energy dissipation inside stilling basin to reduce outgoing velocity and totally solve the common hydraulic problems in large unit discharge and deep tail water projects.
\end{abstract}

\section{Introduction}

The seasonal rainfall requires the low head hydraulic structures to urgently response and increase discharge volume, necessarily raise up tail water depth inside stilling basin and prone to scour downstream river bed due to insufficient energy dissipation and large outgoing velocity outside stilling basin ${ }^{[1-2]}$. Recently, chute sill blocks or baffle blocks are installed above the stilling basin slab to enhance turbulent dissipation and improve flow field. But compared to tail water depth, auxiliary energy dissipators are so small in sizes that almost couldn't touch the mainstream or improve flow field inside stilling basin. Worsely, large underflow velocity inside stilling basin is prone to induce cavitation or some other secondary problems to auxiliary energy dissipators $^{[3-4]}$.

Combined with so many low head hydraulic projects, literature 2 pointed out that flaring gate pier (FGP) would remarkably promote turbulent energy dissipation and shorten stilling basin length. Through shrinking flow section in overflow weir chamber, longitudinal high speed water jet with 3 exposure faces would take shape to continually entrain air in the process of falling down in air and violently impinge on downstream tail water inside stilling basin, which would significantly aggravate turbulent energy dissipation and collision. But referred to previous engineering practices, FGP among overflow weir should meet unique operation requirements. In order to guarantee discharge capacity for overflow weir and reserve enough room for the running down water jet, the ration of downstream water head inside stilling basin to upstream total water head should meet some requirements, which almost confine FGP in application within high water head dams. FGP is almost never seen in low head hydraulic projects for the limited running space in air for water jet.

Many researchers have proposed different optimization measures on FGP through model test, numerical simulation or prototype observation to expand FGP application domain. Yin ${ }^{[5]}$ progressively studied the relationship between hydraulic jump length, height and flow energy ratio in $3 \mathrm{D}$ hydraulic jump through model test. $\mathrm{Li}^{[6-7]}$ indicated FGP with some large convergence angle could promote water jet to longitudinally stretch and reduce impingement pressure on stilling basin slab in high arch dam projects. $\mathrm{Li}^{[8]}$ changed FGP contraction ratio and pointed out the influence of water head upstream overflow weir and FGP contraction ratio on discharge capacity. Zhang ${ }^{[9]}$ comparatively studied the hydraulic characteristics of X-shaped and Y-shaped FGP with RNG k- $\varepsilon$ turbulent model, and emphasized that Xshaped FGP may not only contain the characteristics of air entrainment and water diffusion of conventional Yshaped FGP, but also increase overflow weir discharge capacity and improve pressure distribution along overflow weir surface. $\mathrm{Hu}^{[10]}$ further analyzed the influence factors of aerated cavity in stepped overflow weir combined with FGP through standard k- $\varepsilon$ two equation turbulent model based on Suo Fengyin Hydropower.

\footnotetext{
* Corresponding author: Zhou Zhao, doctor candidate, 1021235166@qq.com
} 
Currently, the engineering application or research about FGP are mainly focused on large unit discharge and high water head project, and the arrangement of FGP is conventional that every chamber is symmetrically installed with FGP. But none researchers have questioned the FGP arrangement and application domain, meanwhile, FGP is almost never applied in large unit discharge and low head projects. In view of previous researcher's omission, this paper modifies the FGP arrangement and proposes incompletely FGP (shown in figure 1-a and below paragraph would call such FGP arrangement as incompletely FGP). Making full use of lateral width of overflow weir and installing FGP in interval chamber, incompletely FGP could aggravate velocity gradients and water level gap between rushing inflow from adjacent chamber. Combined with RNG k- $\varepsilon$ turbulent model and VOF method, this paper would detailedly present the flow pattern, flow field and velocity distribution inside stilling basin.

\section{Engineering practice}

The discharge structure of such hydropower is composed of 5 surface-outlet overflow weir, and each chamber is marked as $1 \#, 2 \#, \ldots \ldots .5 \#$ from left to right bank with corresponding longitudinal chamber axis as $1-1 \ldots . .5-5$

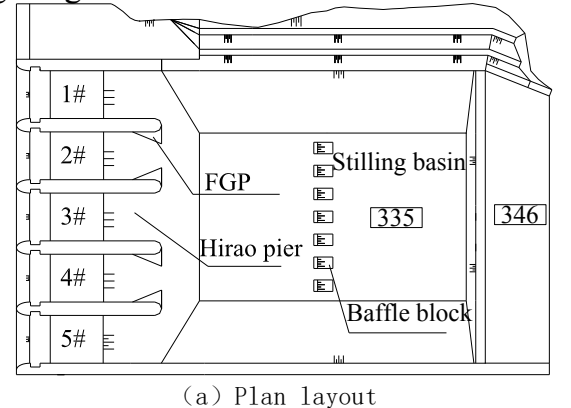

Figure 1 Arrangement for incompletely FGP program (unit:m)

Notification: The unit for above elevation and stake number is $\mathrm{m}$, and $0+00$ is lateral axis of overflow weir.

\section{Numerical model}

Aimed at the full turbulent development inside stilling basin, this paper particularly chooses the most widely used RNG $\mathrm{k}-\varepsilon$ two-equation turbulent model in current engineering practices and adopts commercially available CFD package, Flow-3D, to divide 3D solid model with hexahedral mesh. The unique mesh processing technology, called FAVOR, is adopted in Flow-3D to evaluate the mesh quality and VOF method to be adopted to trace free surface in the process of numerical simulation

\subsection{Governing equations}

General governing equations for incompressible fluid are Reynolds-averaged Navier-equations. And the continuity equation, momentum equation, turbulence kinetic energy (TKE)equation, turbulence energy dissipation rate equation are respectively displayed as following. in this paper. The elevation of overflow weir crest reaches $364.5 \mathrm{~m}$ with net width of inflow section reaching $5 \times 12.5 \mathrm{~m}$. Downstream overflow weir is followed by $1: 1$ fold line underflow stilling basin. The reservoir inflow of check condition and design condition respectively reaches $13200 \mathrm{~m}^{3} / \mathrm{s} 、 9600 \mathrm{~m}^{3} / \mathrm{s}$ with corresponding unit discharge up to $211.2 \mathrm{~m}^{2} / \mathrm{s}, 153.6 \mathrm{~m}^{2} / \mathrm{s}$. Upstream check flood level and design flood level respectively reaches $387 \mathrm{~m}, 383 \mathrm{~m}$, and corresponding downstream water level inside stilling basin reaches $381.64 \mathrm{~m}, 375.43 \mathrm{~m}$, only $5.39 \mathrm{~m}, 7.57 \mathrm{~m}$ lower than upstream water level. Aimed at intensifying energy dissipation and improving flow field inside stilling basin in such low water head project of typical characteristics of large unit discharge, this paper respectively arranges two different programs to comparatively study $3 \mathrm{D}$ flow field inside stilling basin. Program 1 is symmetrically arranged with 5 flat gate piers from $1 \#$ to $5 \#$ chamber, and program 2, call incompletely FGP, is respectively arranged with 2 flaring gate piers in $2 \#, 4 \#$ chamber, while $1 \#, 3 \#, 5 \#$ chamber is flat gate pier. The contraction ration of FGP is 0.5 , and the longitudinal contraction angle is $22.25^{\circ}{ }^{[11]}$. The top of FGP reaches $375.72 \mathrm{~m}$ so as to prevent rushing outflow to inundate FGP. Incompletely FGP is shown as Figure 1 .

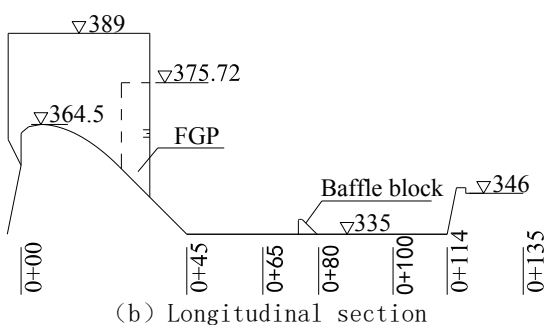

b) Longitudinal section

$$
\begin{gathered}
\frac{\partial \rho}{\partial t}+\frac{\partial \rho u_{i}}{\partial x_{i}}=0 \\
\frac{\partial\left(\rho u_{i}\right)}{\partial t}+\frac{\partial\left(\rho u_{i} u_{j}\right)}{\partial x_{j}}=-\frac{\partial p}{\partial x_{i}}+\frac{\partial}{\partial x_{j}}\left[\left(\mu+\mu_{t}\right)\left(\frac{\partial u_{i}}{\partial x_{j}}+\frac{\partial u_{j}}{\partial x_{i}}\right)\right] \\
\frac{\partial(\rho k)}{\partial t}+\frac{\partial\left(\rho k u_{i}\right)}{\partial x_{i}}=\frac{\partial}{\partial x_{j}}\left[\sigma_{k}\left(\mu+\mu_{t}\right) \frac{\partial k}{\partial x_{j}}\right]+G_{k}-\rho \varepsilon \\
\frac{\partial(\rho \varepsilon)}{\partial t}+\frac{\partial\left(\rho \varepsilon u_{i}\right)}{\partial x_{i}}=\frac{\partial}{\partial x_{j}}\left[\sigma_{\varepsilon}\left(\mu+\mu_{t}\right) \frac{\partial \varepsilon}{\partial x_{j}}\right]+C_{\varepsilon 1}^{*} \frac{\varepsilon}{k} G_{k}-C_{\varepsilon 2} \rho \frac{\varepsilon^{2}}{k}
\end{gathered}
$$

where $\rho, \mu$ are volume fraction average density and molecular viscosity, respectively. $p$ denotes the pressure acting on fluid element. The $\mu \mathrm{t}$ stands for turbulence viscosity and it could be calculated by the turbulence kinetic energy $\mathrm{k}$ and turbulence energy dissipation rate $\varepsilon$. $\mathrm{Gk}$ is generate item of turbulence kinetic energy due to mean velocity gradients.

$$
\begin{aligned}
& \mu_{t}=\rho C_{u} \frac{k^{2}}{\varepsilon} \\
& G_{k}=\mu_{t}\left(\frac{\partial u_{i}}{\partial x_{j}}+\frac{\partial u_{j}}{\partial x_{i}}\right) \frac{\partial u_{i}}{\partial x_{j}}
\end{aligned}
$$




$$
\begin{aligned}
& C_{\varepsilon 1}^{*}=C_{\varepsilon 1}-\frac{\eta\left(1-\eta / \eta_{0}\right)}{1+\beta \eta^{3}} \\
& \eta=\left(2 E_{i j} \bullet E_{\mathrm{ij}}\right)^{0.5} \frac{\mathrm{k}}{\varepsilon} \\
& E_{i j}=\frac{1}{2}\left(\frac{\partial u_{i}}{\partial x_{j}}+\frac{\partial u_{j}}{\partial x_{i}}\right)
\end{aligned}
$$

where $\mathrm{Cu}=0.0845$ is an experimental constant. The turbulence Plandtl numbers for $\mathrm{k}$ and $\varepsilon$ are all 1.39, respectively. And $\mathrm{C} \varepsilon_{1}=1.42, \mathrm{C}_{2}=1.68, \eta_{0}=4.377$, and $\beta=0.012$ are constants for $\varepsilon$ equation.

\subsection{Free surface tracking}

The VOF method is a kind of means to trace free surface variation along overflow weir, which evolves from the marker-and-cell method but more computationally efficient. Recently, Hirt ${ }^{[12]}$, Savage ${ }^{[13]}$ et al introduced VOF method in detail.

VOF method believes both kinds of fluid wouldn't interpenetrate and its main idea is to utilize a variable $F$ to account for how many percentages of each computational cell is occupied by the phase. The variable $\mathrm{F}$ is called volume fraction of the phase and is applied to equation $10 . \mathrm{F}=0$ means there is no liquid in control volume, and $\mathrm{F}=1$ means liquid occupies the full control volume. Furthermore, $\mathrm{F}=0 \sim 1$ means control volume is free liquid surface and partly filled with liquid.

$$
\frac{\partial F}{\partial t}+u \frac{\partial F}{\partial x}+v \frac{\partial F}{\partial y}+w \frac{\partial F}{\partial z}=0
$$

\subsection{Mesh divide}

This calculation model is related with large regions and complicated structure. In order to accurately trace hydraulic characteristic in the control volume, a large scale of hexahedron grids are used to divide the computation domain. Total number of grid cells reaches $8.80 \mathrm{E}+06$ in which $4.70 \mathrm{E}+06$ are active. Considering the differences between upstream and downstream of overflow weir, the numerical domain is divided into three mesh blocks (shown in figure 2). Mesh block is particularly compressed with a uniform mesh size $\triangle X=$ $\triangle \mathrm{Y}=\triangle \mathrm{Z}=0.5 \mathrm{~m}$ among overflow weir and stilling basin regions. Upstream reservoir region and downstream natural channel region are all chosen with uniform mesh size with $\triangle X=\triangle Y=\triangle Z=1 \mathrm{~m}$. The Cartesian coordinate system is used with $\mathrm{X}, \mathrm{Y}, \mathrm{Z}$ representing streamwise, lateral direction and wall-normal, respectively. $\mathrm{X}$ axis directs to the right bank along lateral overflow weir axis, $\mathrm{Y}$ axis directs to streamwise along longitudinal overflow weir axis, and $\mathrm{Z}$ axis is opposite to gravity.

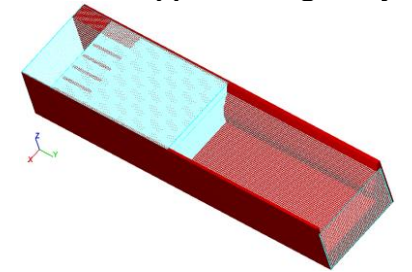

Fig.2 Schematic model meshing

\subsection{Boundary condition and initial condition}

To accurately simulate the given flow, the boundary condition of numerical model should be as similar as possible to what is actually occurring. There are six different boundaries in simulated domain among the Cartesian coordinate. Boundary conditions are set as following: sidewalls x-common no slip, non-porous wall; top z-pressure boundary with gauge pressure equal to zero (atmospheric),bottom z- no slip wall; left y- local stagnation pressure based on upstream total head over overflow weir crest with a hydrostatic pressure distribution, right $y$ - local static pressure based on downstream tailwater with a hydraulic pressure distribution. 50m upstream and 400m downstream of overflow weir are used as boundaries of left and right $\mathrm{Y}$, respectively.

\section{Result and analysis}

\subsection{Model verification}

In view of difference and symmetry in overflow weir chamber of each program, longitudinal water level along the axis of $2 \#, 3 \#$ chamber from numerical simulation and measured values is particularly chosen to verify the numerical simulation accuracy. The results of water level along each chamber axis are shown in figure 3, and numerical data are quite close to practical measured values. There are only some slight discrepancy in upper region of stilling basin where rushing inflow violently fluctuates. Such results can indicate the numerical simulation to be reliable.

Further, the discharge volume of flat gate pier program in check condition and design condition respectively reaches $13820 \mathrm{~m} 3 / \mathrm{s} 、 10040 \mathrm{~m} 3 / \mathrm{s}$, while the discharge volume of incompletely FGP program reaches $13540 \mathrm{~m} 3 / \mathrm{s} 、 9860 \mathrm{~m} 3 / \mathrm{s}$. Both program all can meet the requirement of flood discharge. Comprehensively comparing above discharge volume in two crucial conditions, discharge capacity of incompletely FGP program is only $2.0 \% 、 1.7 \%$ lower than that of the flat gate pier program respectively, indicating such arrangement of incompletely FGP is of no influence to discharge capacity for overflow weir.

\subsection{Flow pattern}

Due to deep tail water inside stilling basin, the submerged hydraulic jump refluxes much and almost inundates overflow weir crest in flat gate pier program. There is almost no fluctuation or lateral diffusion inside the total stilling basin, but outgoing flow greatly upwells right at the end of stilling basin in view of baffle sill. Water level of outgoing flow reaches up high to $378.66 \mathrm{~m}$ with the stable secondary hydraulic jump $30 \mathrm{~m}$ downstream stilling basin. Above visible flow pattern indicates energy dissipation inside stilling basin is seriously insufficient and outgoing flow still carries much redundant energy. Similarly, rushing inflow from 
$1 \#, 3 \#, 5 \#$ flat gate pier chamber forms a visible submerged hydraulic jump. Yet, in view of shrunken flow section in 2\#,4\# FGP chamber, water jet with high speed heavily impinges on tail water and greatly diffuses towards both side due to velocity gradients and water level gap between adjacent rushing flow, forming evident V-shaped intersection with much spilt entrapped bubble and intensifying shear turbulence in upper region of stilling basin. Rushing inflow from bilateral 1\#,5\#

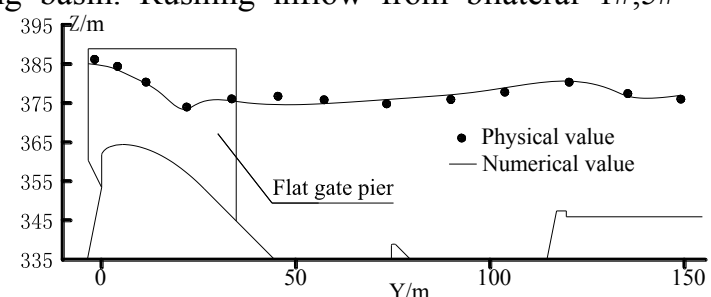

a Water level along longitudinal axis of $2 \#$ chamber in flat gate pier

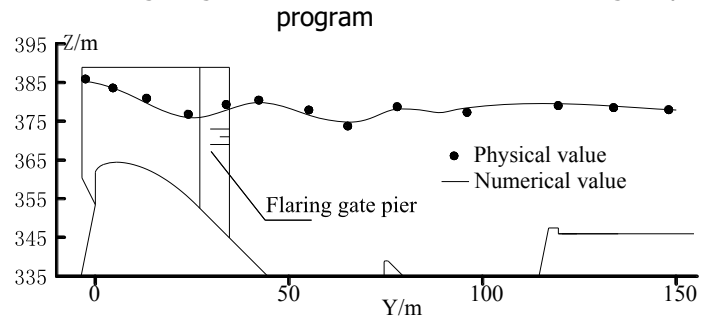

c Water level along longitudinal axis of $2 \#$ chamber in incompletely FGP program chamber is also forced to offset and violently rub guide wall on both sides, inducing symmetrical large scale vertical vortexes from bottom to surface. In such program, momentum interchange and energy transfer are observably enhanced, so does the energy dissipation inside stilling basin. Outgoing flow smoothly joins downstream natural tail water and secondary hydraulic jump outside stilling basin is totally eliminated.

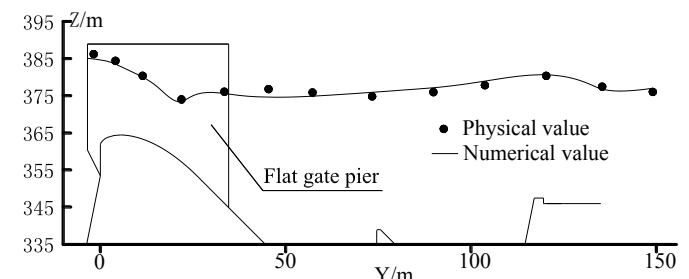

b Water level along longitudinal axis of $3 \#$ chamber in flat gate pier program

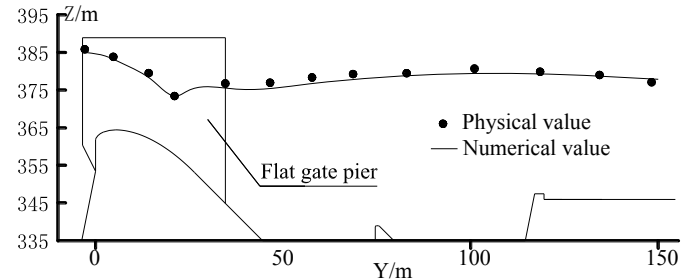

d Water level along longitudinal axis of $3 \#$ chamber in incompletely FGP program

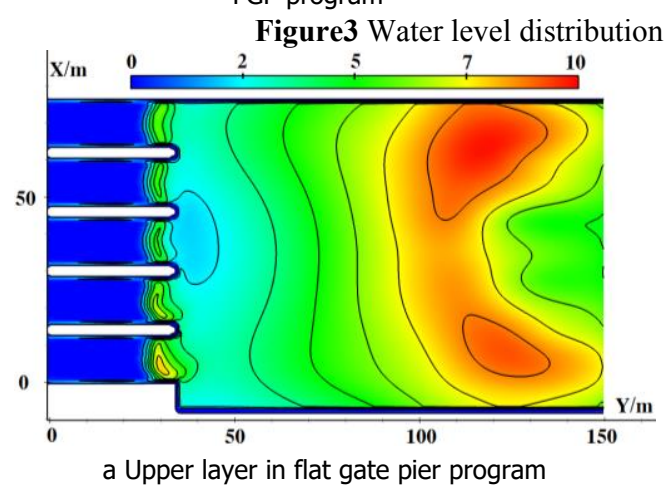

in program

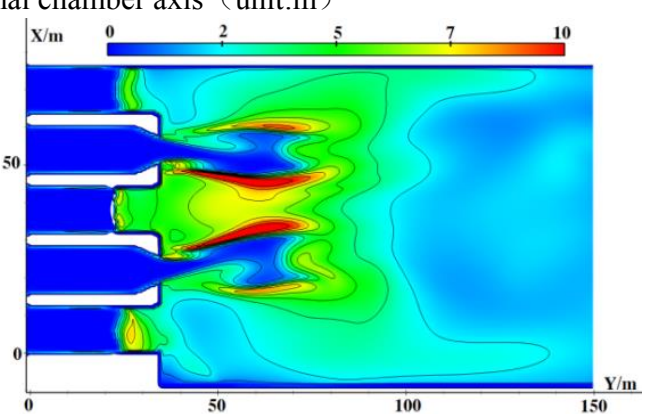

b Upper layer in incompletely FGP program

\subsection{Turbulent kinetic energy distribution}

Turbulence kinetic energy $\left(\mathrm{T}_{\mathrm{KE}}\right)$ is an important kind of physical quantity weighting the extent of running flow fiercely moving, and $\mathrm{T}_{\mathrm{KE}}$ would greatly moves up where discharge flow fiercely dissipates with turbulence. Combined with numerical calculation, $\mathrm{T}_{\mathrm{KE}}$ of discharge flow inside stilling basin can been calculated with formula $T_{K E}=\left(\overline{\mu_{x}^{\prime 2}}+\overline{\mu_{y}^{\prime 2}}+\overline{\mu_{z}^{\prime 2}}\right) / 2$, where $\overline{u_{x}{ }^{\prime}}, \overline{u_{y}{ }^{\prime}}$ and $\overline{u_{z}{ }^{\prime}}$ are mean value of pulsating flow rate in lateral, streamwise and anti-gravity direction, respectively.

Shown in figure 4 , the turbulent kinetic energy of both programs differs much in upper layer of discharge flow. Rushing inflow from 5 flat gate pier chambers in program 1 evenly impinges on tail water and there is a serious lack of enough turbulence in deep layer of running flow. Maximum turbulent kinetic energy only reaches $6 \mathrm{~m}^{2} / \mathrm{s}^{2}$ in upstream the stilling basin, while outgoing flow reaches up high to $10 \mathrm{~m}^{2} / \mathrm{s}^{2}$ above the baffle sill $(0+120 \mathrm{~m})$, consistent with the secondary hydraulic jump in above described flow pattern. But stribution inside stilling basin (unit: $\mathrm{m}^{2} / \mathrm{s}^{2}$ )

turbulence kinetic energy distribution is upper layer in incompletely FGP program is quite from that of flat gate pier program. Shown in figure 4-b, turbulent kinetic energy distribution can exactly indicate rushing inflow from FGP chamber strongly collides with that from flat gate chamber and forms 4 observable high energy turbulent areas on both side of water jet, indicating that energy dissipation and turbulent dissipation are quite significant in upstream the stilling basin due to velocity gradients and water level gap between adjacent rushing inflow. Maximum turbulent kinetic energy reaches $12.5 \mathrm{~m}^{2} / \mathrm{s}^{2}$ and turbulent regions concentrate in upstream the stilling basin. Turbulent kinetic energy rapidly drops down and values of outgoing flow is as low as $2.2 \mathrm{~m}^{2} / \mathrm{s}^{2}$, or even more lower, similarly consistent with stable flow pattern in above described in incompletely FGP program.

\subsection{Aeration concentration distribution}

Water jet with 3 exposed faces from FGP chamber is bound to aerate much air during the process of falling down and entrained bubble is inevitably sucked into 
rushing water to prevail in the whole stilling basin. Squeezed by uneven pulsating pressure from nearby running water, entrained bubble would repeat to collapse, break and shatter into smaller bubble, which would aggravate and compel rushing flow to randomly spread to intensify irregular turbulence and shear friction ${ }^{[14-15]}$.

The longitudinal entrance section $(0+45 \mathrm{~m})$ and middle section $(0+70 \mathrm{~m})$ in stilling basin are particularly chosen to present aeration concentration distribution. Figure 5-a,5-b both indicates rushing flow aerates little and aeration concentration is as low as 0.15,0.20, respectively. Worse, aerated involved depth is seriously inadequate, only reaching $25 \mathrm{~m}$ below free surface, and bottom flow almost finds none evident bubble. Yet, aeration concentration of each corresponding section in incompletely FGP program generally reaches 2 times or more than that of program 1 and aerated scope is much

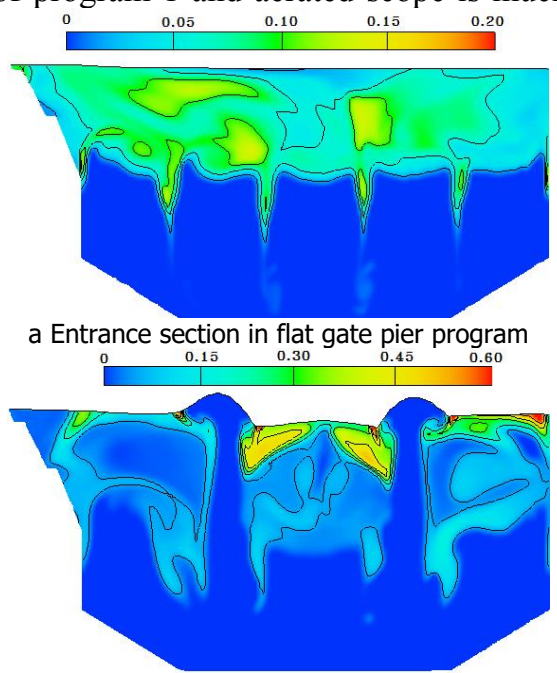

c Entrance section in incompletely FGP program

Figure 5 Aeration concentration in each section inside stilling basin

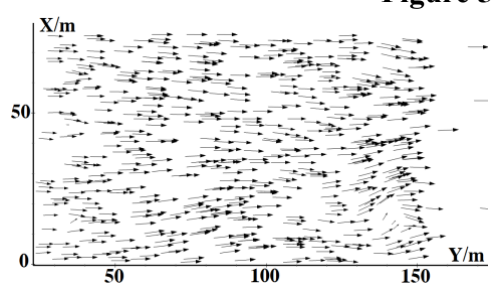

a $Z=355$ elevation in flat gate pier program

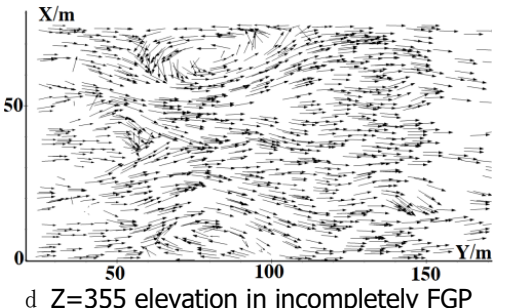

d $Z=355$ elevation in incompletely FGP program

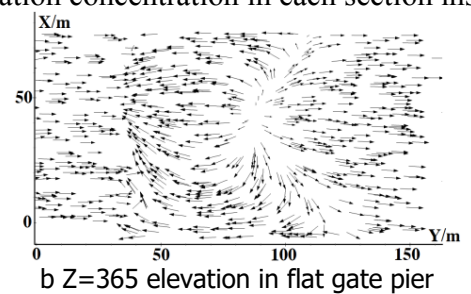

program

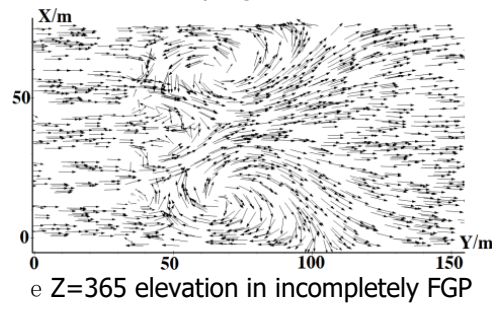

program enlarged, too. Maximum aeration concentration in entrance section reaches up high to 0.6 , or more, and $38 \mathrm{~m}$ below free surface is also involved with aeration concentration high up to 0.15 . There is only slight lack of aerated bubble just downstream the rushing inflow from $2 \#, 4 \#$ chamber due to the highly shrunk and stretched water jet in such regions, further indicating lateral diffusion is strongly related to aeration. Meanwhile, with rushing flow fully offsetting and diffusing, aeration concentration in middle section $(0+70 \mathrm{~m})$ distributes much more evenly and widely. Aeration concentration of the total middle layer running flood in such section generally reaches up high to 0.15 , and even $33 \mathrm{~m}$ below free surface could observe large scale bubble, which is far more massive than that of program 1.

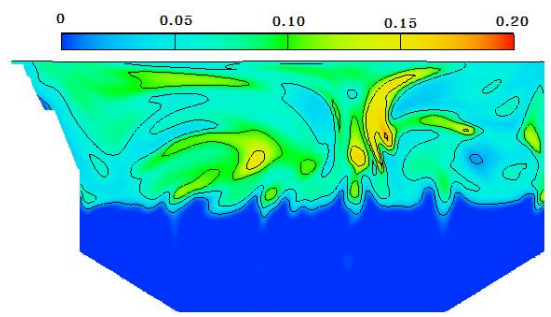

b Middle section in flat gate pier program

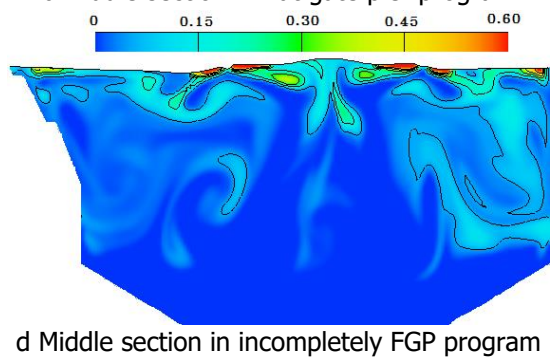

Figure 6 Schematic diagram of horizontal flow field inside stilling basin

\subsection{Horizontal flow field inside stilling basin}

Horizontal flow field is of much importance to trace trajectory of running flow inside stilling basin to reveal interaction between adjacent inflow. In view of longitudinal stream layer deviation due to submerged hydraulic jump, bottom $(Z=355 \mathrm{~m})$, middle $(\mathrm{Z}=365 \mathrm{~m})$, surface $(Z=375 \mathrm{~m})$ flow field are particularly presented to reveal how rushing flow of each layer diffuses. There are no observable differences among rushing flow from each chamber in program 1, and both bottom and middle layer inflow longitudinally impinges on tail water while upper layer inflow violently refluxes, forming submerged hydraulic jump and large scale lateral axis vortex at $0+65 \mathrm{~m}$ section. Such flow field between adjacent rushing inflow can indicates that the flat gate pier program can only depend on conventional submerged 
hydraulic jump to kill superfluous energy, but actually inadequate due to deep tail water inside stilling basin. Obstructed by baffle sill at the end of stilling basin, outgoing flow observably upwells and there are evident surface reflux inside the whole stilling basin. But rushing inflow from each chamber in incompletely FGP program differs much. Inflow from $1 \#, 3 \#, 5 \#$ is particularly similar to that of program 1 , only submerged hydraulic jump slightly moves upstream to $0+50 \mathrm{~m}$ and lateral axis vortex dives deeper. Meanwhile, high speed stretched water jet from 2\#,4\# FGP chamber, shown in figure 6d,6-e,6-f, greatly diffuses and offset towards both sides, inducing vertical vortex to aggravate shear turbulence in view of velocity gradients between adjacent rushing inflow. Little scale vertical vortex is observed near $0+80 \mathrm{~m}$ section among bottom rushing flow field. Further, large scale vertical vortex grows and expands to involve the region from $0+50 \mathrm{~m}$ section to $0+80 \mathrm{~m}$ section among middle and upper rushing flow field, indicating shear turbulence to be gradually remarkable from bottom to surface in upstream regions of stilling basin.

Comprehensively comparing the difference between two programs, what can be found is that baffle blocks are so small in size relative to tail water depth inside stilling basin that almost has no influence on flow field. But installing FGP in interval chamber could observably result in large scale symmetrical vertical vortex on both side of stilling basin from bottom to surface to aggravate shear turbulence for adjacent rushing inflow in upstream the stilling basin due to enlarged lateral velocity gradients and water level gap between adjacent rushing inflow from each chamber.

\subsection{Velocity distribution and energy dissipation rate}

Velocity distribution of check condition in both programs is shown in table 1, in which rushing inflow of program 1 is relatively much larger with maximum velocity in entrance section reaching up high to $13.3 \mathrm{~m} / \mathrm{s}$ and surface reflux reaching as low as $-2.7 \mathrm{~m} / \mathrm{s}$. Energy dissipation inside stilling basin is of much insufficient along the longitudinal stream, underflow velocity is as much as $10-12 \mathrm{~m} / \mathrm{s}$ and maximum outgoing flow velocity downstream stilling basin reaches $12 \mathrm{~m} / \mathrm{s}$ with average velocity $5.87 \mathrm{~m} / \mathrm{s}$. While due to large scale vertical vortex on both side of stilling basin in incompletely FGP program, rushing inflow velocity observably decreases down to $10.1 \mathrm{~m} / \mathrm{s}$ with reflux velocity as high as $-4 \mathrm{~m} / \mathrm{s}$. In view of diving main stream and lateral diffusion, rushing flow velocity of middle layer or upper layer swiftly decreases, so does the outgoing flow. Maximum velocity of outgoing flow decreases down to $6.8 \mathrm{~m} / \mathrm{s}$ with average velocity $4.44 \mathrm{~m} / \mathrm{s}$, which is $24 \%$ lower than of flat gate pier program.

Energy dissipation rates of both programs are shown in table 2, in which the most traditional calculation formula for energy dissipation rate $\eta=\left(E_{1}-E_{2}\right) / E_{1} \times 100 \%$ is chosen. E1、E2 means total water head for running section in upstream the overflow weir and downstream stilling basin, and contains potential energy, pressure energy and kinetic energy. Overflow weir crest section and stilling basin outlet section are particularly adopted for above calculation, and horizontal plane of $Z=347.5 \mathrm{~m}$ is chosen as reference surface for potential energy. Due to high water level inside stilling basin, potential energy accounts for a large proportion for rushing flow and kinetic energy is in relatively small proportion, leading to calculation results for energy dissipation rate to be much little. Energy dissipation rates in check condition for both programs only reach $21.09 \%, 25.85 \%$, respectively, and design condition to be $26.61 \%, 30.21 \%$. In both conditions energy dissipation rate for incompletely FGP program is $4 \%$ higher than that of flat gate pier program, and such progress is of much improvement in large unit discharge and low head project.

Table 1 Velocity distribution along longitudinal section inside stilling basin (unit:m/s)

\begin{tabular}{|c|c|c|c|c|c|c|c|c|c|c|}
\hline Program & $\begin{array}{c}\text { Stake } \\
/ \\
(\mathrm{m})\end{array}$ & bottom & $\begin{array}{c}\text { Left } \\
\text { section } \\
\text { middle }\end{array}$ & surface & bottom & $\begin{array}{l}\text { Middle } \\
\text { section } \\
\text { middle }\end{array}$ & surface & bottom & $\begin{array}{c}\text { Right } \\
\text { section } \\
\text { middle }\end{array}$ & surface \\
\hline \multirow{6}{*}{$\begin{array}{l}\text { Flat gate } \\
\text { pier }\end{array}$} & $0+45$ & 9 & 12.6 & -2.7 & 6.3 & 13.1 & -0.9 & 8.1 & 13.3 & -1.8 \\
\hline & $0+65$ & 12.8 & 9 & -2.7 & 10.8 & 12.6 & -0.9 & 11.7 & 8.8 & -1.8 \\
\hline & $0+80$ & 13.5 & 4.5 & -2.7 & 10.8 & 9 & -0.9 & 12.6 & 5.4 & -2.7 \\
\hline & $0+100$ & 12.5 & 3.8 & 2.3 & 3.8 & 10.5 & -1.5 & 11.3 & 5.3 & -2.3 \\
\hline & $0+114$ & 5.8 & 7.5 & 1.5 & 4.5 & 10.5 & 1.5 & 7.5 & 9.8 & 0.8 \\
\hline & $0+135$ & 2.3 & 11.3 & 3.8 & 0.8 & 11.3 & 4.5 & 3 & 12 & 3.8 \\
\hline \multirow{6}{*}{$\begin{array}{c}\text { Incompletely } \\
\text { FGP }\end{array}$} & $0+45$ & 9.8 & 12 & -4.4 & 9.4 & 7 & -4.4 & 10.1 & 5.5 & -3.1 \\
\hline & $0+65$ & 12.5 & 2.3 & -3.1 & 10.9 & 3.9 & -3.1 & 12.5 & 3.1 & -3.9 \\
\hline & $0+80$ & 12.5 & -2.3 & -2.3 & 6.2 & 5.5 & 3.9 & 11.7 & -3.1 & -3.9 \\
\hline & $0+100$ & 10.1 & 3.1 & -1.6 & 1.6 & 7 & 3.9 & 9.4 & 2.3 & 1.6 \\
\hline & $0+114$ & 3.1 & 5.5 & 2.3 & 1.8 & 7 & 3.9 & 3.6 & 7.3 & 2.7 \\
\hline & $0+135$ & 1.9 & 6.3 & 4.6 & 1.2 & 6.8 & 5.5 & 2.4 & 6.7 & 4.6 \\
\hline
\end{tabular}

Notification: Left, middle, and right section are respectively along longitudinal axis of $1 \#, 3 \#$ and $5 \#$ chamber. 
Table 2 Energy dissipation in each condition

\begin{tabular}{ccccccc}
\hline Condition & Program & $\begin{array}{c}\text { Velocity } \\
\text { above } \\
\text { overflow } \\
\text { weir } \\
\text { crest(m/s) }\end{array}$ & $\begin{array}{c}\text { potential } \\
\text { energy } \\
\text { above } \\
\text { overflow } \\
\text { weir crest } \\
(\mathrm{m})\end{array}$ & $\begin{array}{c}\text { Outgoing } \\
\text { velocity } \\
\text { outside stilling } \\
\text { basin }(\mathrm{m} / \mathrm{s})\end{array}$ & $\begin{array}{c}\text { potential } \\
\text { energy } \\
\text { outside } \\
\text { stilling basin } \\
(\mathrm{m})\end{array}$ & $\begin{array}{c}\text { energy } \\
\text { dissipation } \\
\text { rate }\end{array}$ \\
\hline \multirow{2}{*}{ Check } & $\begin{array}{c}\text { Flat gate pier } \\
\text { Incompletely } \\
\text { FGP }\end{array}$ & 10.07 & 36.67 & 5.87 & 31.26 & $21.09 \%$ \\
\hline \multirow{2}{*}{ Design } & 10.43 & 37.15 & 4.44 & 30.65 & $25.85 \%$ \\
\hline & $\begin{array}{c}\text { Flat gate pier } \\
\text { Incompletely } \\
\text { FGP }\end{array}$ & 9.22 & 33.39 & 5.51 & 26.14 & $26.61 \%$ \\
\hline
\end{tabular}

\section{Conclusion}

Aimed at the common hydraulic problems of secondary hydraulic jump and river bed erosion in deep tail water projects due to insufficient energy dissipation inside stilling basin, this paper proposes a new kind of FGP arrangement-incompletely FGP to improve flow pattern and drop down outgoing velocity. The mechanism of incompletely FGP is revealed with numerical simulation and conclusions are below:

1 The new arrangement of incompletely FGP almost has no much influence on discharge capacity for overflow weir, only $2 \%$ lower than that of conventional flat gate pier program.

2 High speed water jet with 3 exposed faces would observably aerate and spread in the process of running down in air, necessarily enhancing aeration concentration and involved depth. Aeration concentration of each section is as much as 2 times than that of conventional flat gate pier program.

3 Shrinking lateral flow section in partial chamber in incompletely FGP program, high speed water jet from FGP chamber would observably offset and diffuse in upstream of stilling basin due to great velocity gradients and water level gap between adjacent inflow from flat gate pier chamber, inducing large scale vertical vortex from bottom to surface on both side and aggravating shear turbulence inside stilling basin. Energy dissipation effect is improved and secondary hydraulic jump outside stilling basin never appears again.

Comprehensively considering discharge capacity, flow pattern, aeration concentration and horizontal flow field inside stilling basin, what can be found is that the new arrangement of incompletely FGP not only guarantees discharge capacity, enhance aeration concentration and intensify shear turbulence or vertical vortex inside stilling basin with great velocity gradients, but also totally solves the secondary hydraulic jump with insufficient energy dissipation.

\section{Reference}

1. Xie Shengzong, Zhu Ronglin, Li Shiqing, et al. Hydraulics of bucket basin equipped with flaring gate piers[J], Journal of hydraulic engineering, 2:7$18,(1992)$
2. Xie Shengzong, Wu Yihong, Chen Wenxue. New technology and innovation on flood discharge and energy dissipation of high dams in China[J], Journal of hydraulic engineering, 47(3):324-336,(2016)

3. An Gang, Yuan Ximin, Yang Chanzeng, et al. The experimental studyof energy dissipation structure of low water head project[J]. Journal of Tianjin University, 30(4):500-506 (1997)

4. Yang Fan, Wang Junxing, Chen Shuai, et al. Study on cavitation damages of supplementary dissipater under low-Froude-number flows[J], Journal of hydroelectric engineering, 34(2):125-129,(2015)

5. Yin Jinbu, Liang Zongxiang, Gong Honglin. Experimental study on flow characteristics of 3D hydraulic jump of flaring gate piers[J], Journal of hydraulic engineering,41(11):1333-1338,(2011)

6. Li Naiwen, Xu Weilin, Tianzong, et al. Optimal experimental study on flaring piers of surface spillways in high arch dam[J], Journal of hydroelectric engineering, 28(3):132-138,(2009)

7. Li Naiwen, Liu Chao, Deng Jun,etal. Theoretical and experimental studies of the flaring gate pier on the surface spillway in a high-arch dam[J]. Journal of Hydrodynamics. 24(4): 496-505,(2012)

8. Li Futian, Liu Peiqing, $\mathrm{Xu}$ Weilin, et al. Experimental study on effect of flaring piers on weir discharge capacity in high arch dam[J], Journal of hydraulic engineering, 11:43-47(2003)

9. Zhang Ting, Wu Chao, Mo Zhengyu, et al. Comparison on hydraulic characteristics of $\mathrm{X}$-shape and Y-shape flaring gate piers[J], Journal of hydraulic engineering, 38(10):1207-1213,(2007)

10. Hu Yaohua, Wu Chao, Zhang Ting, et al. Affect factors analysis of aerated cavity of X-shaped flaring gate pier[J], Journal of Sichuan University (engineering science edition), 39(3):24-28, (2007)

11. Li Zhongshu, Pan Yanhua, Han Lianchao, et al. Hydraulics of stilling basin with flaring gate piers[J], Advance in water science, 11(1):82-87,(2000)

12. Hirt C W, Nichols B D. Volume of fluid (VOF) method for the dynamics of free boundarues[J].J Compus Phys, 39(3):201-225,(1981)

13. Savage, B.M; Johnson, M.C, Flow over ogee spillway: Physical and numerical model case 
study[J], Journal of hydraulic engineering, 127(8):640-649,(2001)

14. Liao Liuxia, Wang Wei, Liu Shanjun, et al. Influence of local sidewalls placed behind flaring gate pier on aeration effectiveness[J]. Journal of
Sichuan University (engineering science edition), 46(2):13-16,(2014)

15. Deng Jun, Xu Weilin, Velocity distribution of selfaerated flows[J] Journal of hydrodynamics, 17(3):369-375,(2002) 